Acta Crystallographica Section B

Structural

Science

ISSN 0108-7681

Pamela A. McGregor, ${ }^{\text {a David R. }}$ Allan, ${ }^{\mathrm{a} *}$ Simon Parsons ${ }^{\mathrm{a}}$ and Stewart J. Clark ${ }^{b}$

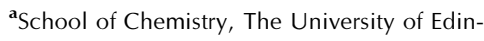
burgh, Edinburgh EH9 3JJ, Scotland, and

${ }^{\mathbf{b}}$ Department of Physics, The University of Durham, Science Laboratories, South Road, Durham DH1 3LE, England

Correspondence e-mail: d.r.allan@ed.ac.uk

\title{
Hexamer formation in tertiary butyl alcohol (2-methyl-2-propanol, $\mathrm{C}_{4} \mathrm{H}_{10} \mathrm{O}$ )
}

The crystal structure of phase II of tertiary butyl alcohol (2-methyl-2-propanol, $\mathrm{C}_{4} \mathrm{H}_{10} \mathrm{O}$ ) has been solved using a combination of single-crystal X-ray diffraction techniques and $a b$ initio density functional calculations. This trigonal $P \overline{3}$ phase, which is stable at both low temperature and high pressure, and the triclinic $P \overline{1}$ phase (phase IV) have very similar enthalpies, the calculations revealing only a $3.859 \mathrm{~kJ} \mathrm{~mol}^{-1}$ enthalpy difference at ambient pressure, despite the substantial change of the intermolecular bonding motif from helical catemer to hexamer with an increase in pressure or reduction in temperature. The hexamers in the trigonal phase adopt a chair conformation. There are two unique hexamers: at low temperature these are centred at $(0$, $\left.0, \frac{1}{2}\right)$ and $\left(\frac{2}{3}, \frac{1}{3}, 0.961(13)\right)$, and at high pressure the centres are $\left(0,0, \frac{1}{2}\right)$ and $\left(\frac{2}{3}, \frac{1}{3}, 0.958(14)\right)$. A slight flattening of the hexamers is observed at high pressure and the calculations confirm that phase II becomes more stable relative to phase IV on pressure increase.

\section{Introduction}

The study of the structural systematics of small-molecule systems is rewarding as they reveal trends in whole classes of compounds and larger molecular systems. Brock \& Duncan (1994) have described the general features of the packing motifs adopted by mono-alcohols $(R \mathrm{OH})$ where there is a competition between the packing requirements of the relatively bulky $R$ group and the need for the small hydroxyl groups to be close enough to form hydrogen bonds. If the molecules are relatively small then they can form catemers, generated by either a glide plane or a $2_{1}$-screw axis, where the molecules form an approximately coplanar alternating sequence about the central chain of hydrogen bonds. If the molecules contain larger $R$ groups, steric hindrance often prohibits this simple arrangement and, instead, the molecules often form chains about three-, four- or sixfold screw axes, or adopt crystal structures with more than one molecule in the asymmetric unit. Molecules containing particularly bulky $R$ groups may no longer form hydrogen-bonded chains or catemers, but cyclic dimer, trimer, tetramer or hexamer rings can be created. We have been investigating the high-pressure polymorphism of a range of prototypical monoalcohols including methanol (Allan et al., 1998), ethanol (Allan \& Clark, 1999; Allan et al., 2001), phenol (Allan et al., 2002) and its halogenated derivatives (e.g. 2-chlorophenol and 4-fluorophenol) (Oswald, Allan, Day et al., 2005; Oswald, Allan, Motherwell \& Parsons, 2005), and, more recently, cyclobutanol (McGregor et al., 2005) and cyclopentanol (Moggach et al., 2005), with a view to establishing trends in their packing and hydrogen-bonding behaviour. In general, we find that
Received 27 October 2005 Accepted 27 April 2006
(C) 2006 International Union of Crystallography Printed in Great Britain - all rights reserved 
pressure tends to transform the packing of the $R$ groups from having characteristics more closely associated with bulky groups to those more typical of small groups: i.e. pressure tends to promote catemers with a simple alternating sequence of molecules rather than the formation of helical chains or rings. Here we have extended our range of studies to tertiary butyl alcohol ( $t$-butanol, $\mathrm{C}_{4} \mathrm{H}_{10} \mathrm{O}$ ), which has the bulkiest $R$ group of all the monoalcohol systems we have investigated to date.

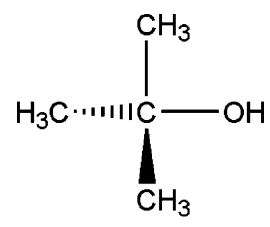

The low-temperature behaviour of $t$-butanol in the solid state has been studied using a variety of techniques, including calorimetry, spectroscopy, neutron scattering and X-ray diffraction (Steininger et al., 1989). In the calorimetric measurements of Oetting (1963), three crystalline phases were noted, although their structures were not determined. It was found that phase II is stable below $281 \mathrm{~K}$ and, from their IR studies, Sciesinska \& Sciesinska (1980) concluded that the crystal structure contains hydrogen-bonded chains of molecules. Phase II was found to transform into either phase I at $286.14 \mathrm{~K}$ or phase III at $281.54 \mathrm{~K}$; the latter was reported to have a milky appearance and was stable between approximately 282 and $295 \mathrm{~K}$. Although Steininger et al. (1989) reported that it is difficult to grow single crystals of phase III, they found that needle-like single crystals of phase I could be formed readily if the melt was supercooled in the temperature range between $281 \mathrm{~K}$ and the melting point at $298.97 \mathrm{~K}$. The IR work of Sciesinska \& Sciesinska (1980) indicates that the

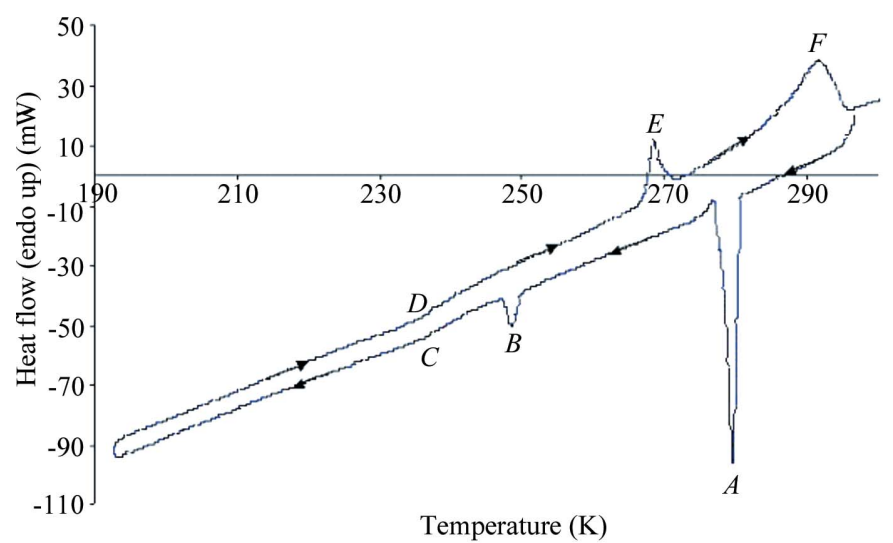

Figure 1

Graph of heat flow versus temperature for a DSC experiment on $t$-butanol. The sample was loaded as a liquid at room temperature. The temperature was scanned from 298 to $193 \mathrm{~K}$ and then cycled back to $313 \mathrm{~K}$ at a rate of $10 \mathrm{~K} \mathrm{~min}{ }^{-1} . A=$ crystallization, $B=$ phase transition from phase IV to phase II, $C, D=$ anomaly due to a possible ordering of the methyl groups, $E=$ phase transition from phase II to phase IV, $F=$ melting. structure of phase I is likely to be formed from 'curl-like' disordered hydrogen-bonded chains (Steininger et al., 1989). Dilatometric studies by Neu (1968) show that a further phase of $t$-butanol exists as the density of a sample of phase I increases with time if it is stored at $298 \mathrm{~K}$.

Additionally, Atkins (1911) followed a similar annealing process and observed that the needle-like crystals of phase I, which are nucleated from the supercooled liquid, are transformed into crystals with a rhombohedral habitus (phase IV) if they are held at a temperature close to the melting point. Despite the prominence of $t$-butanol in the monoalcohol series, its wide use in chemistry as a solvent and its rich phase behaviour, it is only this phase, phase IV, that has so far had its structure determined (Steininger et al., 1989; CSD refcode VATSAK). Steininger et al. (1989) used single-crystal X-ray diffraction techniques on a zone-refined sample to show that phase IV of $t$-butanol has a triclinic structure with the space group $P \overline{1}$ and that it is characterized by hydrogen-bonded helical chains of molecules arranged along the $a$ axis of the unit cell. There are two of these helices in the unit cell (each composed of three symmetry-independent molecules) and they are related by the inversion centre.

Here we report the first crystal structure determination of phase II of $t$-butanol, at both low-temperature and highpressure, where we have used a combination of single-crystal $\mathrm{X}$-ray diffraction techniques and $a b$ initio density-functional calculations. We find that the structure is trigonal with $P \overline{3}$ symmetry and is characterized by the formation of hydrogenbonded hexamers. Although the structure of phase II is very different from that of phase IV, the calculations indicate that the alteration of the hydrogen bonding of the molecules from catemer to hexamer results in a relatively small increase in the enthalpy (3.859 $\mathrm{kJ} \mathrm{mol}^{-1}$ ) for the trigonal structure at ambient pressure. At $0.85 \mathrm{GPa}$, the difference in enthalpy between the two crystal structures is reversed (and increases in magnitude slightly to $13.314 \mathrm{eV}$ per molecule), indicating that only a fairly modest pressure is required to make the structure of phase II the relatively more stable.

\section{Experiment and ab initio calculations}

Initially, the low-temperature phase behaviour of $t$-butanol was surveyed by differential scanning calorimetery (DSC) measurements. These results were obtained using a Perkin Elmer Pyris DSC 1. The sample of $t$-butanol was distilled and dried using a vacuum line and loaded as a liquid into a sealed aluminium pan. The procedure was carried out at a scan rate of $10 \mathrm{~K} \mathrm{~min}^{-1}$ from 293 to $193 \mathrm{~K}$ and then back to room temperature (Fig. 1).

Crystallization occurred at $280.6 \mathrm{~K}$ and was indicated by the large exothermic peak (event $A$ in Fig. 1). The peak also has a small endothermic shoulder at $276.5 \mathrm{~K}$. The next event is at $250 \mathrm{~K}$ on cooling (event $B$ ), where another exothermic change occurs, due to a structural phase transition. This is followed by another event at $238 \mathrm{~K}$ where there is a slight kink in the graph on both cooling and heating (events $C$ and $D$ ). It is postulated that this feature is due to ordering in the sample - perhaps due 
Table 1

Refinement statistics for the low-temperature $(220 \mathrm{~K})$ and high-pressure $(0.85 \mathrm{GPa})$ structure determinations of the trigonal $P \overline{3}$ phase (phase II) of tertiary butanol.

\begin{tabular}{|c|c|c|}
\hline & Low temperature & High pressure \\
\hline \multicolumn{3}{|l|}{ Crystal data } \\
\hline Chemical formula & $\mathrm{C}_{4} \mathrm{H}_{10} \mathrm{O}$ & $\mathrm{C}_{4} \mathrm{H}_{10} \mathrm{O}$ \\
\hline$M_{r}$ & 74.12 & 74.12 \\
\hline $\begin{array}{l}\text { Cell setting, space } \\
\text { group }\end{array}$ & Trigonal, $P \overline{3}$ & Trigonal, $P \overline{3}$ \\
\hline Temperature (K) & $220(2)$ & $293(2)$ \\
\hline$a, c(\AA)$ & $18.0946(13), 8.4041(9)$ & $17.55(2), 8.080(10)$ \\
\hline$V\left(\AA^{3}\right)$ & 2383.0 & $2155(4)$ \\
\hline$Z$ & 18 & 18 \\
\hline$D_{x}\left(\mathrm{Mg} \mathrm{m}^{-3}\right)$ & 0.930 & 1.028 \\
\hline Radiation type & Mo $K \alpha$ & Mo $K \alpha$ \\
\hline $\begin{array}{l}\text { No. of reflections for } \\
\text { cell parameters }\end{array}$ & 3699 & 16 \\
\hline$\theta$ range $\left({ }^{\circ}\right)$ & $2.5-25$ & $2-10$ \\
\hline$\mu\left(\mathrm{mm}^{-1}\right)$ & 0.06 & 0.07 \\
\hline Crystal form, colour & Cylinder, colourless & Cylinder, colourless \\
\hline Crystal size $(\mathrm{mm})$ & $0.5 \times 0.1 \times 0.1$ & $0.02 \times 0.02 \times 0.01$ \\
\hline \multicolumn{3}{|l|}{ Data collection } \\
\hline Diffractometer & Bruker SMART APEX & Enraf-Nonius CAD-4 \\
\hline $\begin{array}{l}\text { Data collection } \\
\text { method }\end{array}$ & $\omega$ scans & $\omega$ scans \\
\hline Absorption correction & $\begin{array}{l}\text { Multi-scan (based on } \\
\text { symmetry-related } \\
\text { reflections) }\end{array}$ & $\begin{array}{l}\text { Multi-scan (based on } \\
\text { symmetry-related } \\
\text { reflections) }\end{array}$ \\
\hline$T_{\min }$ & 0.816 & 0.253 \\
\hline$T_{\max }$ & 1.000 & 0.562 \\
\hline $\begin{array}{l}\text { No. of measured, } \\
\text { independent and } \\
\text { observed reflections }\end{array}$ & $12157,2818,2217$ & $1808,1203,550$ \\
\hline $\begin{array}{l}\text { Criterion for observed } \\
\text { reflections }\end{array}$ & $I>2 \sigma(I)$ & $I>2 \sigma(I)$ \\
\hline$R_{\text {int }}$ & 0.026 & 0.211 \\
\hline$\theta_{\max }\left({ }^{\circ}\right)$ & 25.0 & 20.0 \\
\hline Range of $h, k, l$ & $\begin{array}{l}-21 \Rightarrow h \Rightarrow 21 \\
-21 \Rightarrow k \Rightarrow 21 \\
-10 \Rightarrow l \Rightarrow 4\end{array}$ & $\begin{array}{l}0 \Rightarrow h \Rightarrow 13 \\
-16 \Rightarrow k \Rightarrow 14 \\
-4 \Rightarrow l \Rightarrow 7\end{array}$ \\
\hline \multicolumn{3}{|l|}{ Refinement } \\
\hline Refinement on & $F^{2}$ & $F^{2}$ \\
\hline $\begin{array}{l}R\left[F^{2}>2 \sigma\left(F^{2}\right)\right], w R\left(F^{2}\right) \\
\quad\end{array}$ & $0.044,0.131,1.07$ & $0.115,0.295,1.01$ \\
\hline No. of reflections & 2818 & 1203 \\
\hline No. of parameters & 144 & 64 \\
\hline H-atom treatment & $\begin{array}{l}\text { Constrained to parent } \\
\text { site }\end{array}$ & $\begin{array}{l}\text { Constrained to parent } \\
\text { site }\end{array}$ \\
\hline Weighting scheme & $\begin{array}{l}w=1 /\left[\sigma^{2}\left(F_{o}^{2}\right)+\right. \\
\left.\quad(0.0783 P)^{2}+0.0635 P\right] \\
\text { where } \\
\quad P=\left(F_{o}^{2}+2 F_{c}^{2}\right) / 3\end{array}$ & $\begin{array}{l}w=1 /\left[\sigma^{2}\left(F_{o}^{2}\right)+\right. \\
\left.\quad(0.1552 P)^{2}\right], \text { where } \\
P=\left(F_{o}^{2}+2 F_{c}^{2}\right) / 3\end{array}$ \\
\hline$(\Delta / \sigma)_{\max }$ & 0.164 & 0.014 \\
\hline$\Delta \rho_{\max }, \Delta \rho_{\min }\left(\mathrm{e} \AA^{-3}\right)$ & $0.13,-0.13$ & $0.33,-0.21$ \\
\hline Extinction method & SHELXL97 & None \\
\hline Extinction coefficient & $0.0000(12)$ & - \\
\hline
\end{tabular}

to the ordering of the methyl groups. The X-ray diffraction data were collected at $220 \mathrm{~K}$ and we have assigned the phase in this temperature region as phase II to be consistent with the results of Oetting (1963), who found that phase II is stable below $281 \mathrm{~K}$.

On heating from $193 \mathrm{~K}$ to room temperature, the kink in the scan at $235 \mathrm{~K}$ is observed, then an endothermic event at $267.3 \mathrm{~K}$ (event $E$ ). This is the phase change associated with the exothermic peak at $250 \mathrm{~K}$ (i.e. event $B$ ) on cooling, although there is some hysteresis observed in the sample. The melting curve of the sample is very shallow; the onset is just above the phase transition at $270 \mathrm{~K}$ and is completed some $20 \mathrm{~K}$ later at $290 \mathrm{~K}$ (event $F$ ). This is the region where Oetting (1963) identified three further phases in the sample; it is clear from this experiment that there are no further phase transitions above $268 \mathrm{~K}$, although there may have been a mixture of solid and liquid which may have affected their experiments. Consequently, we tentatively assign events $B$ and $E$ to being due to a phase transition between phase II and phase IV.

\subsection{Low-temperature study}

A distilled sample of $t$-butanol was loaded into a capillary using a vacuum line in order to prevent exposure to air and therefore reduce the risk of contamination of water into the hygroscopic sample. The capillary, with an internal diameter of $\sim 0.1 \mathrm{~mm}$, was mounted and centred on a Bruker SMART APEX diffractometer (Siemens, 1993; graphite monochromated Mo $K \alpha$ radiation) equipped with a cryogenic cooling system, initially set at $243 \mathrm{~K}$, and a $25 \mathrm{~W}$ OHCD laserassisted crystal grower (Boese \& Nussbaumer, 1994). This initial temperature ensured that the polycrystalline sample was safely in the phase II region. Subsequently, the temperature was raised to just below the phase II to phase IV transition temperature, and the laser was used to establish a solidliquid boundary. A single crystal was obtained by zone refinement of the sample over a time span of $20 \mathrm{~min}$. A series of frames was collected both to assess the crystal quality and to provide an initial unit cell for the sample at the $268 \mathrm{~K}$ crystal growth temperature. The reflections were indexed using GEMINI (Sparks, 1999), and the crystal system was found to be trigonal. The sample was then cooled to $220 \mathrm{~K}$, whilst monitoring the diffraction pattern every $10 \mathrm{~K}$, to ensure that the event associated with points $C$ and $D$ in the DSC experiment did not degrade the sample quality. A hemisphere of data was collected using $20 \mathrm{~s}$ exposures and $0.3^{\circ}$ scans in $\omega$. This data set was indexed, integrated, solved and refined using GEMINI (Sparks, 1999), SAINT (Siemens, 1995) and the SHELX suite (Sheldrick, 1997). The H atoms were placed in idealized positions and the final refinement statistics are presented in Table 1. ${ }^{\mathbf{1}}$ (Although attempts were made to grow single crystals of phase I, the samples proved to be unstable, even when contained within capillaries, and we therefore limited the scope of this study to the determination of phase II.)

\subsection{High-pressure study}

At ambient conditions $t$-butanol is a crystalline solid, but on gentle heating it can be readily liquefied. The $t$-butanol sample was loaded and pressurized in a Merrill-Bassett diamond anvil cell (Merrill \& Bassett, 1974), which had been warmed to a temperature just above the melting point of $t$-butanol and had been equipped with $600 \mu \mathrm{m}$ culet diamond anvils and a

\footnotetext{
${ }^{1}$ Supplementary data for this paper are available from the IUCr electronic archives (Reference: WS5038). Services for accessing these data are described at the back of the journal.
} 
Table 2

Lattice parameters $\left(\AA,{ }^{\circ}, \AA^{3}\right)$ obtained from the ab initio density functional calculations for both the triclinic $P \overline{1}$ and the trigonal $P \overline{3}$ phases of tertiary butanol at 0 and $0.85 \mathrm{GPa}$.

\begin{tabular}{lll}
\hline & $0 \mathrm{GPa}$ & $0.85 \mathrm{GPa}$ \\
\hline$P \overline{1}$ & & \\
$a$ & 6.2027 & 6.0631 \\
$b$ & 9.1431 & 9.0661 \\
$c$ & 14.7554 & 14.4493 \\
$\alpha$ & 86.373 & 85.248 \\
$\beta$ & 78.776 & 78.531 \\
$\gamma$ & 76.363 & 75.800 \\
$V_{\text {cell }}$ & 797.363 & 754.133 \\
$V_{\text {molecule }}$ & 132.894 & 125.689 \\
$P \overline{3}$ & & \\
$a$ & & 17.4389 \\
$c$ & 17.5898 & 8.0210 \\
$V_{\text {cell }}$ & 8.1014 & 2112.510 \\
$V_{\text {molecule }}$ & 2170.755 & 117.362 \\
\hline
\end{tabular}

tungsten gasket. After the nucleation of many crystallites the temperature was cycled close to the melting curve, in order to reduce the number of crystallites, in a manner similar to that adopted by Vos et al. (1992, 1993). Finally, a single crystal was obtained at approximately 0.85 (1) GPa that entirely filled the $250 \mu \mathrm{m}$ gasket hole.

The setting angles of 17 strong reflections were determined on an Enraf-Nonius CAD-4 diffractometer (equipped with an Mo X-ray tube) and a least-squares fit to the data gave trigonal unit-cell parameters that compare closely with the unit-cell parameters measured at low temperature.

Intensity data were collected with the $\omega$-scan method at the position of least attenuation of the pressure cell, according to the fixed- $\varphi$ technique (Finger \& King, 1978). All accessible reflections were measured in the shell $+h, \pm k, \pm l$ for $0 \AA^{-1}<\sin \theta / \lambda<0.48 \AA^{-1}$. The intensities were corrected for absorption and then used for structure solution by direct methods in $P \overline{3}$ symmetry. Three molecules were identified in the asymmetric unit and the structure was refined with the SHELX suite of programs (Sheldrick, 1997). Owing to the low completeness of the high-pressure data set, the displacement parameters were refined isotropically. The $\mathrm{H}$ atoms were placed in idealized positions. The refinement statistics for the final fit are listed in Table 1.

\section{3. $A b$ initio calculations}

In order to acquire a fuller understanding of the relationship between the low-temperature and high-pressure phases, we have performed a series of $a b$ initio calculations using the CASTEP code (Segall et al., 2002). This also allows us to obtain accurate $\mathrm{H}$-atom positions, which are difficult to obtain using X-ray diffraction techniques.

The calculations were performed using the density functional formalism with the generalized gradient approximation (Perdew \& Wang, 1992) applied for the many electron exchange and correlation interactions. This approach is known to improve the description of the structural and electronic properties of hydrogen-bonded systems compared with the commonly used local density approximation (Perdew \& Zunger, 1981). Non-local ultra-soft pseudopotentials generated by the method of Vanderbilt (1990) were used to describe electron-ion interactions. The valence electron wavefunctions were expanded in a plane-wave basis set with a kinetic energy cut-off of $540 \mathrm{eV}$. This converges the total energy of the system to better than $0.001 \mathrm{eV}$ per molecule $\left(0.096 \mathrm{~kJ} \mathrm{~mol}^{-1}\right)$. Brillouin zone integrations were performed on a Monkhorst-Pack grid (Monkhorst \& Pack, 1976) that is large enough to reach a level of convergence in total energy similar to the wavefunction cut-off. The electronic structure calculation proceeds via a preconditioned conjugate gradients, energy-minimization scheme (Payne et al., 1992) and density mixing algorithm using the plane-wave coefficients as variational parameters.

The experimentally determined atomic positions and lattice parameters were used in the calculations as a starting point, from which relaxed structural parameters were determined. The Hellmann-Feynman theorem was used to calculate the forces on the individual atoms, which were used to relax the structure. The $a b$ initio stresses on the cell were also used to relax the cell parameters. We include a correction to the stresses and total energy (Francis \& Payne, 1990) that is required since the basis set changes as the unit cell is optimized. However, our basis set is large enough that these corrections are very small and are only included for completeness.

\section{Results and discussion}

On performing the calculations to fully relax the structures of phase II and phase IV of $t$-butanol, we find that the lattice parameters are in good agreement with the experimental data (see Table 2). In the calculations, the symmetry of the structures was not constrained and we find that no symmetry breaking occurs during the calculations, indicating that they are in agreement with the experimentally determined space groups for both phases. The calculated fractional coordinates, including those for the $\mathrm{H}$ atoms, are also in excellent agreement with those determined experimentally (see deposited data). This gives us additional confidence that we have accurately determined the structure of phase II from the X-ray diffraction experiments and we have confirmed the structure of phase IV.

The pseudo-threefold nature of the molecular chains in phase IV of $t$-butanol is readily observed in an $a$-axis projection of the crystal structure, as shown in Fig. 2. Neighbouring chains are aligned alternately antiparallel to one another along the $a$ axis and, in graph-set notation, these adopt a $C_{3}^{3}(6)$ configuration. The structure has three unique molecules in the asymmetric unit, which gives rise to three unique hydrogen bonds. The donor-acceptor distances, calculated from the structure reported by Steininger et al. (1989), are $2.714 \AA$ for $\mathrm{O} 1 \cdots \mathrm{O} 3,2.712 \AA$ for $\mathrm{O} 3 \cdots \mathrm{O} 2$ and $2.741 \AA$ for $\mathrm{O} 2 \cdots \mathrm{O} 1(1+$ $x, y, z)$.

In phase II there are three symmetry-independent molecules and these form six-membered rings or hexamers, as shown in Fig. 3. In graph-set notation, both rings have the 
notation $R_{6}^{6}(12)$, with six hydrogen bonds forming each ring. There are two unique hexamers within the structure, referred to as hexamer I and hexamer II. Hexamer I has one unique hydrogen bond and is centred at $\left(0,0, \frac{1}{2}\right)$. Hexamer II has two unique hydrogen bonds and is centred at $\left(\frac{2}{3}, \frac{1}{3}, 0.961\right.$ (13)) in the low-temperature structure and at $\left(\frac{2}{3}, \frac{1}{3}, 0.958(14)\right)$ in the high-pressure structure. Hexamer I lies on a threefold rotoinversion centre, whereas hexamer II lies only on a threefold axis. Both hexamer I and hexamer II are arranged in the 'chair' conformation common to cyclohexane and its derivatives. Neighbouring $t$-butanol molecules are orientated either above or below the mean plane of the ring. Fig. 4 shows the packing arrangement of the hexamers within the phase II crystal structure in projection down the crystallographic $c$ axis. Like phase IV of $t$-butanol, the asymmetric unit of phase II also contains three molecules, which again gives rise to three unique hydrogen bonds within the structure. The donoracceptor distances for the low-temperature structure determination are 2.775 (1) $\AA$ for $\mathrm{O} 1-\mathrm{O} 2(x, y, z+1), 2.779$ (1) $\AA$ for $\mathrm{O} 2-\mathrm{O} 1(-y+1, x-y, z-1)$ and 2.729 (1) $\AA$ for O3-

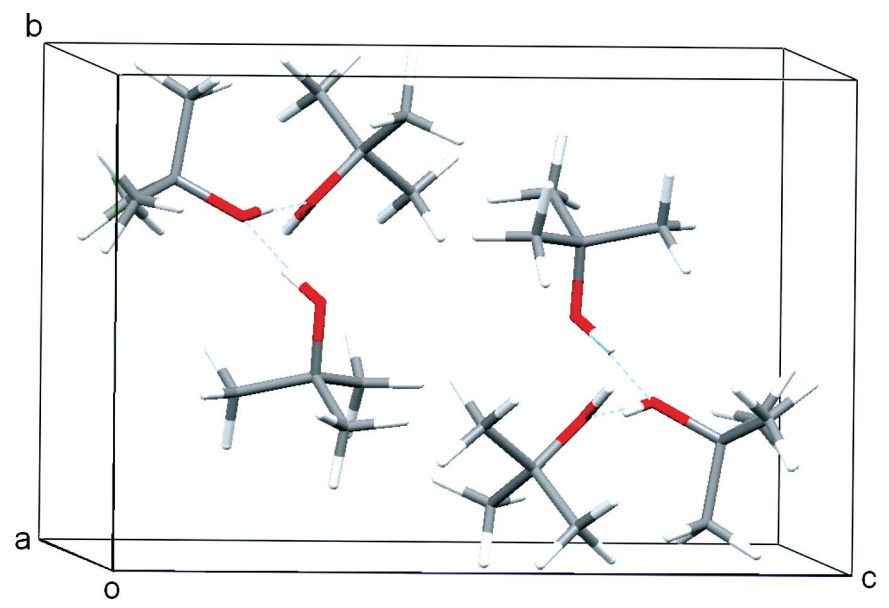

Figure 2

The crystal structure of the triclinic $P \overline{1}$ phase IV of $t$-butanol, viewed approximately along the crystallographic $a$ axis. The threefold helical nature of the hydrogen-bonded molecular chains is apparent. The figure was generated using the unit-cell parameters and fractional coordinates from the $0 \mathrm{GPa}$ calculation.

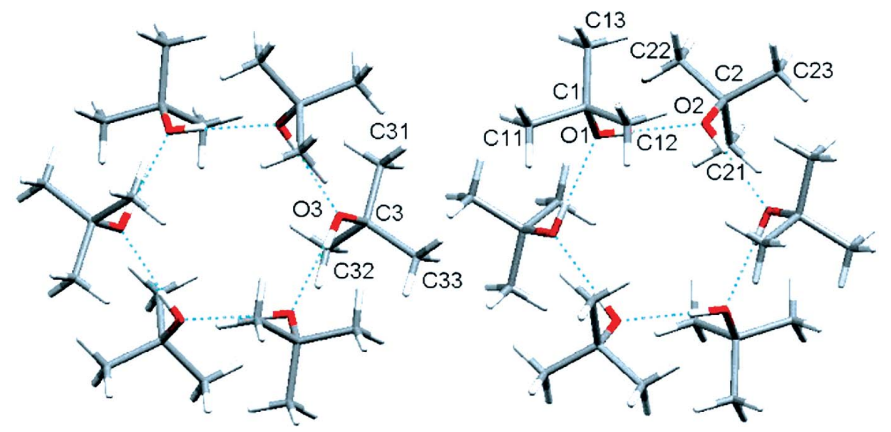

Figure 3

A projection along the crystallographic $c$ axis of hexamer I and hexamer II in the trigonal $P \overline{3}$ phase II of $t$-butanol.
O3( $x-y, x,-z+2)$, while those for the high-pressure structure determination are $2.786(9) \AA$ for $\mathrm{O} 1-\mathrm{O} 2(x, y, z+1)$, 2.720 (9) $\AA$ for $\mathrm{O} 2-\mathrm{O} 1(-y+1, x-y, z-1)$ and $2.698(7) \AA$ for $\mathrm{O} 3-\mathrm{O} 3(x-y, x,-z+2)$.

A measure of the flatness of the hexamers can be made by considering the torsion angle between four neighbouring $\mathrm{O}$ atoms in the ring. Hexamers I and II each have a characteristic torsion angle. In hexamer $\mathrm{I}$, the torsion angle $\mathrm{O} 3-\mathrm{O} 3(y, y-x$, $z)-\mathrm{O} 3(y-x,-x, z)-\mathrm{O} 3(-x,-y, 2-z)$ is $50.1(1)^{\circ}$ at $220 \mathrm{~K}$ and reduces to $42.1(7)^{\circ}$ at $0.85 \mathrm{GPa}$. In hexamer II, the torsion angle $\mathrm{O} 1-\mathrm{O} 2(x, y, 1+z)-\mathrm{O} 1(1-y, x-y, z)-$ $\mathrm{O} 2(1-y, x-y, 1+z)$ is found to be $50.1(1)^{\circ}$ at $220 \mathrm{~K}$, which reduces to $47.8(5)^{\circ}$ at $0.85 \mathrm{GPa}$. Hence, with pressure, there is a slight flattening of both hexamers.

Examining the crystal packing as a whole, one notable feature is the range of methyl-methyl contacts present. (All distances refer to the carbon-carbon separations.) Methyl groups on the same $t$-butanol molecule are separated by $2.48 \AA$ on average. The intrahexamer contacts are $3.98 \AA$ for hexamer I, and 4.01 and $4.20 \AA$ for hexamer II. However, the interhexamer separations are significantly shorter, with a shortest methyl-methyl contact of only $3.52 \AA$. The methyl-

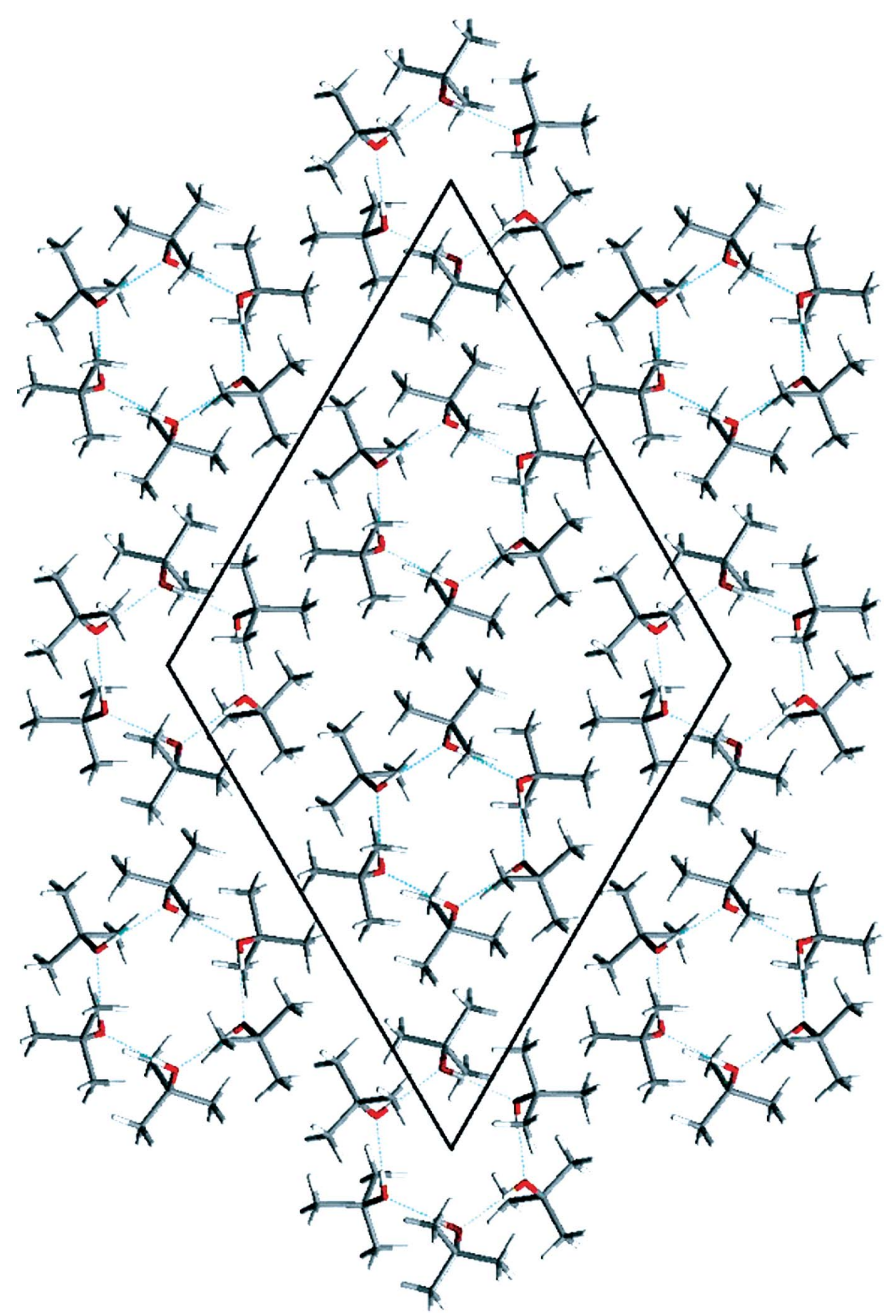

Figure 4

The crystal structure of the trigonal $P \overline{3}$ phase II of $t$-butanol viewed along the crystallographic $c$ axis. 
methyl contact motif is reminiscent of that of the lowtemperature phase of acetic acid as the axes of the interacting methyl groups (defined by the $\mathrm{C}-\mathrm{C}$ bonds) are approximately perpendicular to one another and are directed towards the methyl $\mathrm{C}$ atom of the adjacent interacting molecule (Allan \& Clark, 1999). In the low-temperature phase of acetic acid the methyl-methyl contacts form a zigzag link between adjacent hydrogen-bonded catemers, while the inter-hexamer contacts of the high-pressure phase of $t$-butanol form the vertices of what are almost equilateral triangles arranged on layers parallel to the $x y$ plane (Fig. $5 a$ ). The triangle formed by atoms $\mathrm{C} 13, \mathrm{C} 23$ and C33 is arranged on a layer at $z \simeq \frac{3}{4}$, and the triangle formed by atoms C11, C22 and C31 is formed on a layer $z \simeq \frac{1}{4}$.

In contrast, the interhelical methyl-methyl contact motif of low-temperature $P \overline{1}$ phase IV involves only individual pairs of molecules, on neighbouring helices. The axes defined by the $\mathrm{C}-\mathrm{C}$ bond on each interacting methyl group lie along the line of contact and are almost ideally collinear to one another (Fig. 5b). This form of methyl-methyl interaction motif is very

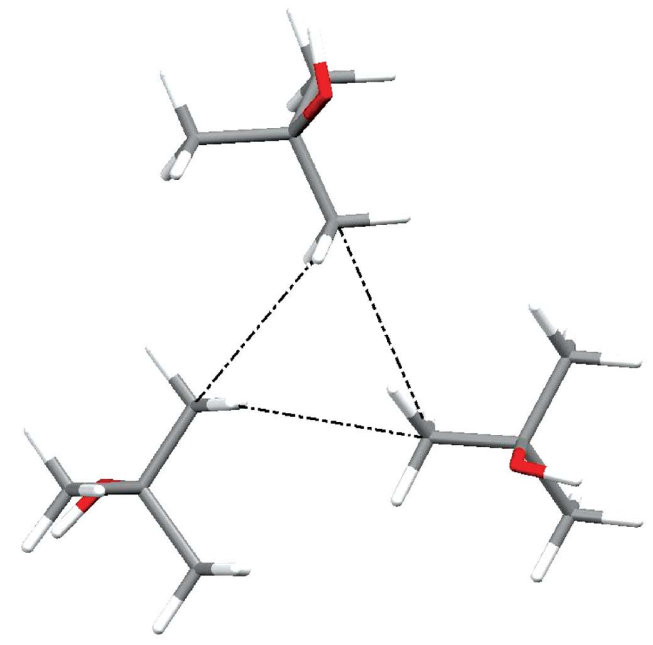

(a)

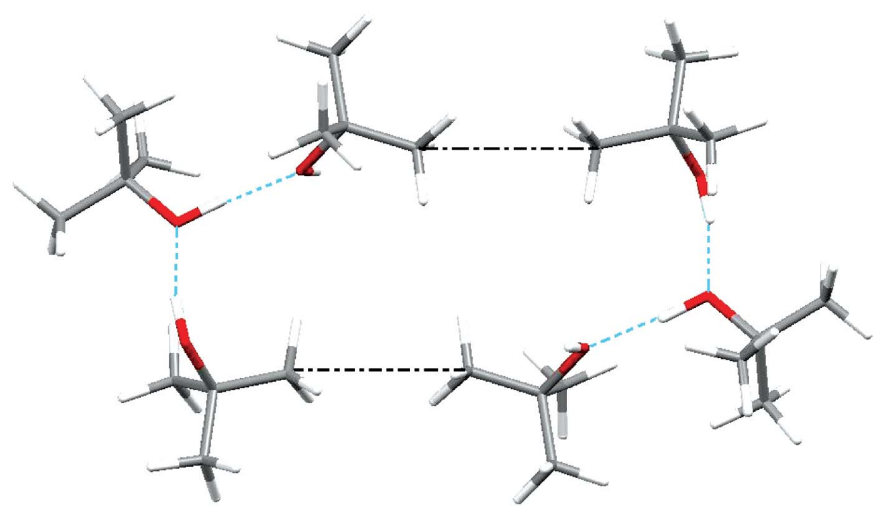

(b)

Figure 5

The methyl-methyl interactions (- - - -) in (a) the trigonal $P \overline{3}$ phase II of $t$-butanol and $(b)$ the triclinic $P \overline{1}$ phase IV of $t$-butanol. similar to that exhibited by the high-pressure phase of acetic acid, where the intercatemer contacts involve only individual pairs of molecules that have almost perfectly coincident molecular axes (Allan \& Clark, 1999). The shortest interhelical methyl-methyl contacts for phase IV of $t$-butanol is $3.67 \AA$, which is significantly shorter than the intrahelical contacts of 4.05, 4.16 and $4.35 \AA$ and marginally longer than the corresponding interhexamer distance in $P \overline{3}$ phase II.

We have been able to examine the relative energies of the phases at both ambient (0.0 GPa) and elevated pressure $(0.85 \mathrm{GPa})$ using the first-principles techniques. The relative total energies per molecule give a measure of the relative stability of each phase, although this approach does not give an indication of barrier heights, which can be used to estimate thermal stability. At ambient pressure it is found that phase IV is more stable than phase II by $3.859 \mathrm{~kJ} \mathrm{~mol}^{-1}$, while at elevated pressure the order of stability is reversed with a difference in enthalpy of $13.314 \mathrm{~kJ} \mathrm{~mol}^{-1}$, indicating that the application of pressure acts to stabilize the trigonal phase II. Our calculations also reveal that phase II is denser than phase IV at both ambient pressure and $0.85 \mathrm{GPa}$ (see Table 2), and this fact may partly explain why we found that phase II would crystallize in preference to phase IV in our ambient-pressure experiments. The difference in the calculated total energies at ambient pressure is certainly relatively small, and the crystallization of one phase in preference to another will strongly depend on the initial nucleation and variations in the crystal growth conditions. These effects, including the influence of crystal seeding on the particular phase grown at ambient pressure, have been documented by Steininger et al. (1989).

Although our calculations reveal that phase II is denser than phase IV at both ambient and elevated pressure (Table 2), our calculations reveal that the difference in relative energy at ambient pressure is fairly modest.

\section{Conclusions}

In conclusion, we have solved the trigonal $P \overline{3}$ phase II crystal structure of tertiary butyl alcohol, at both low-temperature and high-pressure, and find that this phase and the triclinic $P \overline{1}$ phase (phase IV) have very similar enthalpies despite the extremely substantial change of intermolecular bonding motif from helical catemer to hexamer. The hexamers in the trigonal phase adopt a chair conformation and at low temperature these are centred at $\left(0,0, \frac{1}{2}\right)$ and $\left(\frac{2}{3}, \frac{1}{3}, 0.961(13)\right)$, and at high pressure the centres are $\left(0,0, \frac{1}{2}\right)$ and $\left(\frac{2}{3}, \frac{1}{3}, 0.958(14)\right)$. Between these layers, the hexamers are linked by methyl-methyl contacts, which form nearly ideal equilateral triangles on layers at $z \simeq \frac{1}{4}$ and $z \simeq \frac{3}{4}$. The increased stability of phase II over that of phase IV with pressure, as indicated by the series of $a b$ initio calculations, is contrary to the general trend towards small $R$-group behaviour that we have observed in the other members of the monoalcohol series. However, as the pressure range of the current study is extremely limited, we would certainly expect there to be additional polymorphs at higher pressure, which may have crystal structures consisting of relatively simple pseudo-twofold catemers in agreement 
with the general structural trends that we have already observed.

We thank the EPSRC for funding this work and for supporting DRA through his EPSRC Advanced Research Fellowship.

\section{References}

Allan, D. R. \& Clark, S. J. (1999). Phys. Rev. B, 60, 6328-6334.

Allan, D. R., Clark, S. J., Brugmans, M. J. P., Ackland, G. J. \& Vos, W. L. (1998). Phys. Rev. B, 58, R11809-R11812.

Allan, D. R., Clark, S. J., Dawson, A., McGregor, P. A. \& Parsons, S. (2002). Acta Cryst. B58, 1018-1024.

Allan, D. R., Parsons, S. \& Teat, S. J. (2001). J. Synchrotron Rad. 8, 10 17.

Atkins, W. R. G. (1911). J. Chem. Soc. 99, 10-23.

Boese, R. \& Nussbaumer, M. (1994). Correlations, Transformations and Interactions in Organic Chemistry, IUCr Crystallographic Symposia, Vol. 7, edited by D. W. Jones \& A. Katrusiak. Oxford University Press.

Brock, C. P. \& Duncan, L. L. (1994). Chem. Mater. 6, 1307-1312.

Finger, L. W. \& King, H. (1978). Am. Mineral. 63, 337-342.

Francis, G. P. \& Payne, M. C. (1990). J. Phys. Condens. Matter, 2 , 4395-4404.

McGregor, P. A., Allan, D. R., Parsons, S. \& Pulham, C. R. (2005). Acta Cryst. B61, 449-454.

Merrill, L. \& Bassett, W. A. (1974). Rev. Sci. Instrum. 45, 290-294.

Moggach, S. A., Allan, D. R., Parsons, S., Sawyer, L. \& Warren, J. E. (2005). J. Synchrotron Rad. 12, 598-607.
Monkhorst, H. J. \& Pack, J. D. (1976). Phys. Rev. B, 13, 5188-5192. Neu, W. J.-M. (1968). C. R. Acad. Sci. Paris Ser. C, 267, 1025-1028. Oetting, F. L. (1963). J. Phys. Chem. 67, 2757-2761.

Oswald, I. D. H., Allan, D. R., Day, G. M., Motherwell, W. D. S. \& Parsons, S. (2005). Cryst. Growth Des. 5, 1055-1071.

Oswald, I. D. H., Allan, D. R., Motherwell, W. D. S. \& Parsons, S. (2005). Acta Cryst. B61, 69-79.

Payne, M. C., Teter, M. P., Allan, D. C., Arias, T. A. \& Joannopoulos, J. D. (1992). Rev. Mod. Phys. 64, 1045-1097.

Perdew, J. P. \& Wang, Y. (1992). Phys. Rev. B, 45, 13244-13249.

Perdew, J. P. \& Zunger, A. (1981). Phys. Rev. B, 23, 5048-5079.

Sciesinska, E. \& Sciesinska, J. (1980). Acta. Phys. Pol. A, 58, 361368.

Segall, M. D., Lindan, P. J. D., Probert, M. J., Pickard, C. J., Hasnip, P. J., Clark, S. J. \& Payne, M. C. (2002). J. Phys. Condens. Matter, 14, 2717-2744.

Sheldrick, G. M. (1997). SHELXL97. University of Göttingen, Germany.

Siemens (1993). SMART. Siemens Industrial Autom. Inc., Madison, Wisconsin, USA.

Siemens (1995). SAINT. Siemens Industrial Autom. Inc., Madison, Wisconsin, USA.

Sparks, R. A. (1999). GEMINI. Bruker AXS Inc., Madison, Wisconsin, USA.

Steininger, R., Bilgram, J. H., Gramlich, V. \& Petter, W. (1989). Z. Kristallogr. 187, 1-13.

Vanderbilt, D. (1990). Phys. Rev. B, 41, 7892-7895.

Vos, W. L., Finger, L. W., Hemley, R. J., Hu, J. Z., Mao, H. K. \& Schouten, J. A. (1992). Nature (London), 358, 46.

Vos, W. L., Finger, L. W., Hemley, R. J. \& Mao, H. (1993). Phys. Rev. Lett. 71, 3150-3153. 